\title{
Thermodynamic theory for the jamming transition in traffic flow
}

\author{
Takashi Nagatani \\ Division of Thermal Science, College of Engineering, Shizuoka University, Hamamatsu 432-8561, Japan
}

(Received 17 April 1998)

\begin{abstract}
A thermodynamic theory is formulated for describing the phase transition and critical phenomenon occurring in traffic flow. We derive the time-dependent Ginzburg-Landau (TDGL) equation from the car-following model. We find the thermodynamic potential for traffic flow where the headway and the inverse of the delay time correspond respectively to order parameter and temperature. It is shown that the coexisting curve and spinodal line are given respectively by the first and second derivatives of the potential with order parameter (the headway). We prove that the jamming transition is the first-order transition below the critical point and the metastable region exists between the coexisting curve and spinodal line. We show the connection between TDGL equation and the modified Korteweg-de Vries equation describing the traffic jam. We also compare the nonlinear analysis result with the simulation. It is shown that the coexisting curve is consistent with the simulation result. [S1063-651X(98)04710-2]
\end{abstract}

PACS number(s): 05.70.Fh, 05.70.Jk, 89.40.+k

\section{INTRODUCTION}

Recently, traffic problems have attracted considerable attention [1]. A variety of approaches have been applied to describe the collective properties of traffic flow [2-28]. The jamming transitions between the freely moving traffic and the jammed traffic have been found in their traffic models. The transitions have been observed in actual traffic $[29,30]$. The fluid dynamic model and the car-following models have been studied analytically by the linear stability theory and the nonlinear analysis method [31,32]. The modified Korteweg-de Vries (KdV) equation has been derived from the car-following model where traffic jams were described in terms of a kink-antikink solution [32].

The jamming transitions have properties very similar to the conventional phase transition. With increasing car density, the freely moving traffic changes to the jammed traffic at a specific value of density. In the congested traffic flow (at high density), the uniform traffic flow becomes unstable. Then, the unstable traffic flow results in the formation of traffic jams in which the freely moving traffic of low density coexists with the jammed traffic of high density. The freely moving traffic and jammed traffic correspond respectively to the gas and liquid phases in the conventional gas-liquid phase transition: the headway or car density correspond to the volume or density, and the inverse of the delay time (sensitivity parameter) corresponds to temperature. The metastable region appears near the point of maximal current in the fundamental diagram $[33,34]$. The metastability is similar to that in the first-order phase transition. Also, the critical point exists in traffic flow of the car following models $[32,35]$.

Though the properties similar to the phase transitions and critical phenomena have been found in the traffic flow models, to our knowledge, the thermodynamic representation describing the jamming transition was unknown until now. It is important to derive the thermodynamic theory of the traffic flow from the traffic flow models. Also, it is interesting to connect the traffic metastability with the spinodal decomposition in the first-order phase transition.
Now, it is well known that the time-dependent GinzburgLandau (TDGL) equation can describe the nonequilibrium phase transition including the metastability [36,37]. The TDGL equation has two kinds of solutions in the steady state: one is the uniform solution and the other is the solution of the kink-antikink form. Recently, it has been shown that the traffic flow near the critical point can be described by the modified KdV equation which has a traveling wave solution of the kink-antikink form. To our knowledge, how to describe the jamming transition by the TDGL equation has not been known until now.

In this paper, we present a thermodynamic theory to describe the collective properties of traffic flow. We derive the TDGL equation from the car-following model. We find the thermodynamic potential describing the coarse-grained behavior of traffic flow. We prove that the jamming transition and the metastability are described in terms of the thermodynamic theory. We derive the TDGL equation from the modified KdV equation. We carry out the simulation for the car-following model. We compare the theoretical result with the simulation result.

The organization of the paper is as follows. In Sec. II we present a modified version of the car-following model analyzed by Newell [2] and Whitham [3]. In Sec. III we derive the TDGL equation from the car-following model. We give a thermodynamic theory describing the jamming transition. In Sec. IV we derive the modified KdV equation from the carfollowing model. We show the connection between the modified KdV and the TDGL equations. In Sec. V we apply the linear stability analysis to the car-following model. In Sec. IV we present the simulation result. We compare the simulation result with the theoretical result. Section VII gives a summary.

\section{CAR-FOLLOWING MODEL}

We present a modified version of the car-following model analyzed by Newell [2] and Whitham [3]. The car-following model is described by the equation of motion of car $n$ :

$$
d x_{n}(t+\tau) / d t=V\left(\Delta x_{n}(t)\right)
$$


where $\Delta x_{n}\left(=x_{n+1}-x_{n}\right)$ is the headway and $\tau$ is the delay time. The idea is that a driver adjusts the car velocity $d x_{n} / d t$ according to the observed headway $\Delta x_{n}(t)$. The delay time $\tau$ allows for the time lag that it takes the car velocity to reach the optimal velocity $V\left(\Delta x_{n}\right)$ when the traffic flow is varying. Newell [2] and Whitham [3] have used the following optimal velocity function:

$$
V\left(\Delta x_{n}\right)=v_{\max }\left\{1-\exp \left[-(\gamma / v)\left(\Delta x_{n}-L\right)\right]\right\},
$$

where $v_{\max }$ is the maximal velocity, $L$ is the car length, and $\gamma$ is a constant. Equation (2) is a monotonically increasing function with $\Delta x_{n}$. This optimal velocity function does not have the turning point (inflection point). If we choose Newell's function (2) as the optimal velocity, we cannot derive the TDGL equation and the modified KdV equation from Eq. (1). Therefore, we choose the same optimal velocity function as that used by Bando et al. [4]

$$
V\left(\Delta x_{n}\right)=\left(v_{\max } / 2\right)\left\{\tanh \left(\Delta x_{n}-h_{c}\right)+\tanh \left(h_{c}\right)\right\},
$$

where $h_{c}$ is the safety distance. Equation (3) has a turning point at $\Delta x_{n}=h_{c}: V^{\prime \prime}\left(h_{c}\right)=0$. It is important that the optimal velocity function has a turning point. Otherwise, we cannot derive the TDGL equation and the modified KdV equation, which have a kink-antikink density wave solution representing the traffic jam. For simplicity, we set $v_{\max }=2$ hereafter. The realistic values of parameters $v_{\max }$ and $h_{c}$ will be determined by comparing with the observed experimental data of traffic. Bando et al. [4] have determined the values of parameters $v_{\max }$ and $h_{c}$ which reproduce the characteristic features of the observed traffic flow data.

Generally, it is necessary that the optimal velocity function has the following properties: It is a monotonically increasing function, it has an upper bound (maximal velocity), and it has a turning point at the safety distance. Thus, when the headway is less than the safety distance, the car velocity is reduced and small enough to prevent crashing into the preceding car. On the other hand, if the headway is larger than the safety distance, the car moves with higher velocity. The car velocity does not exceed the maximal velocity. Equation (3) satisfies the above properties.

Bando et al. [4] have proposed the optimal velocity model described by the following differential equation:

$$
d^{2} x_{n} / d t^{2}=a\left[V\left(\Delta x_{n}(t)\right)-d x_{n} / d t\right]
$$

where $a$ is the sensitivity and $V\left(\Delta x_{n}\right)$ is given by Eq. (3). In this model, the inverse of sensitivity $a$ corresponds to delay time $\tau$ in Eq. (1). Komatsu and Sasa [32] have derived the modified KdV equation from Eq. (4). Unfortunately, we can not derive the TDGL equation from Eq. (4). Therefore, we adopt the modified Newell's model described by Eq. (1) with Eq. (3).

\section{TDGL EQUATION}

We show the derivation of the TDGL equation from Eqs. (1) and (3). We wish to derive the equation describing the collective motion on coarse-grained scales. We now consider the slowly varying behaviors at long wavelengths. We extract slow scales for space variable $n$ and time variable $t$ [38].
For $0<\varepsilon \ll 1$, we define the slow variables $X$ and $T$,

$$
X=\varepsilon\left(n+V^{\prime} t\right) \text { and } T=\varepsilon^{3} V^{\prime} t,
$$

where $V^{\prime}=d V\left(\Delta x_{n}\right) /\left.d \Delta x_{n}\right|_{\Delta x_{n}=h_{c}}$. We rewrite Eq. (1) as follows:

$$
d \Delta x_{n}(t+\tau) / d t=V\left(\Delta x_{n+1}(t)\right)-V\left(\Delta x_{n}(t)\right) .
$$

We set headway $\Delta x_{n}(t)$ as

$$
\Delta x_{n}(t)=h_{c}+\varepsilon R(X, T) .
$$

By inserting Eq. (7) into the left-hand side of Eq. (6) and expanding to fifth order of $\varepsilon$, one obtains the following:

$$
\begin{aligned}
d \Delta x_{n}(t+\tau) / d t= & \varepsilon^{2} V^{\prime} \partial_{X} R+\varepsilon^{3} V^{\prime 2} \tau \partial_{X}^{2} R+\varepsilon^{4}\left(V^{\prime 3} \tau^{2} / 2\right) \partial_{X}^{3} R \\
& +\varepsilon^{5}\left(V^{\prime 4} \tau^{3} / 6\right) \partial_{X}^{4} R+\varepsilon^{4} \partial_{T} R \\
& +\varepsilon^{5} 2 V^{\prime} \tau \partial_{X} \partial_{T} R
\end{aligned}
$$

By expanding the optimal velocity function around the turning point, inserting Eq. (7) into the right-hand side of Eq. (6), and expanding to fifth order of $\varepsilon$, one obtains

$$
\begin{aligned}
V( & \left.\Delta x_{n+1}\right)-V\left(\Delta x_{n}\right) \\
= & V^{\prime}\left(\Delta x_{n+1}-\Delta x_{n}\right)+\left(V^{\prime \prime \prime} / 6\right)\left[\left(\Delta x_{n+1}-h_{c}\right)^{3}\right. \\
& \left.-\left(\Delta x_{n}-h_{c}\right)^{3}\right] \\
= & V^{\prime}\left[\varepsilon^{2} \partial_{X} R+\varepsilon^{3}(1 / 2) \partial_{X}^{2} R+\varepsilon^{4}(1 / 6) \partial_{X}^{3} R+\varepsilon^{5}(1 / 24) \partial_{X}^{4} R\right] \\
& +\left(V^{\prime \prime \prime} / 6\right)\left[\varepsilon^{4} \partial_{X} R^{3}+\varepsilon^{5}(1 / 2) \partial_{X}^{2} R^{3}\right] .
\end{aligned}
$$

By inserting Eqs. (8) and (9) into Eq. (6), we obtain the following:

$$
\begin{aligned}
\varepsilon^{4} \partial_{T} R+ & \varepsilon^{5} 2 V^{\prime} \tau \partial_{T} \partial_{X} R \\
= & \varepsilon^{3} V^{\prime}\left(1 / 2-V^{\prime} \tau\right) \partial_{X}^{2} R+\varepsilon^{4} V^{\prime}\left(1 / 6-V^{\prime 2} \tau^{2} / 2\right) \partial_{X}^{3} R \\
& +\varepsilon^{4}\left(V^{\prime \prime \prime} / 6\right) \partial_{X} R^{3}+\varepsilon^{5} V^{\prime}\left(1 / 24-V^{\prime 3} \tau^{3} / 6\right) \partial_{X}^{4} R \\
& +\varepsilon^{5}\left(V^{\prime \prime \prime} / 12\right) \partial_{X}^{2} R^{3} .
\end{aligned}
$$

We consider the neighborhood of $V^{\prime} \tau=1 / 2$ :

$$
V^{\prime} \tau=1 / 2+\varepsilon^{2} .
$$

Equation (10) is rewritten as

$$
\begin{aligned}
\varepsilon^{4} \partial_{T} R= & \varepsilon^{4} V^{\prime}(1 / 24) \partial_{X}^{3} R-\varepsilon^{4}\left(\left|V^{\prime \prime \prime}\right| / 6\right) \partial_{X} R^{3} \\
& +\varepsilon^{3} V^{\prime}\left(1 / 2-V^{\prime} \tau\right) \partial_{X}^{2} R-\varepsilon^{5} V^{\prime}(1 / 48) \partial_{X}^{4} R \\
& +\varepsilon^{5}\left(\left|V^{\prime \prime \prime}\right| / 12\right) \partial_{X}^{2} R^{3},
\end{aligned}
$$

where $V^{\prime \prime \prime}<0$.

By transforming variables $X$ and $T$ to variables $x=\varepsilon^{-1} X$ and $t=\varepsilon^{-3} T$ and taking $\varepsilon R(X, T)=S(x, t)$, Eq. (12) is rewritten as follows:

$$
\begin{aligned}
\partial_{t} S= & \left(V^{\prime} / 24\right) \partial_{x}^{3} S-\left(\left|V^{\prime \prime \prime}\right| / 6\right) \partial_{x} S^{3}-V^{\prime}\left(V^{\prime} \tau-1 / 2\right) \partial_{x}^{2} S \\
& -\left(V^{\prime} / 48\right) \partial_{x}^{4} S+\left(\mid V^{\prime \prime \prime} / 12\right) \partial_{x}^{2} S^{3} .
\end{aligned}
$$


By adding term $2 V^{\prime}\left(V^{\prime} \tau-1 / 2\right) \partial_{x} S$ to both the left- and right-hand sides and performing Galilean transformation: $t_{1}$ $=t$ and $x_{1}=x+2 V^{\prime}\left(V^{\prime} \tau-1 / 2\right) t_{1}$, we obtain

$$
\begin{aligned}
\partial_{t_{1}} S= & {\left[\partial_{x_{1}}-(1 / 2) \partial_{x_{1}}^{2}\right]\left[\left(V^{\prime} / 24\right) \partial_{x_{1}}^{2} S+2 V^{\prime}\left(V^{\prime} \tau-1 / 2\right) S\right.} \\
& \left.-\left(\left|V^{\prime \prime \prime}\right| / 6\right) S^{3}\right] .
\end{aligned}
$$

We define the thermodynamic potential:

$$
\phi(S) \equiv-V^{\prime}\left(V^{\prime} \tau-1 / 2\right) S^{2}+\left(\left|V^{\prime \prime \prime}\right| / 24\right) S^{4},
$$

where $V^{\prime}=V^{\prime}\left(h_{c}\right)$ and $V^{\prime \prime \prime}=V^{\prime \prime \prime}\left(h_{c}\right)(<0)$. By rewriting Eq. (14) with Eq. (15), we obtain the TDGL equation:

$$
\partial_{t_{1}} S=-\left[\partial_{x_{1}}-(1 / 2) \partial_{x_{1}}^{2}\right] \delta \Phi(S) / \delta S
$$

with

$$
\Phi(S) \equiv \int d x\left[\left(V^{\prime} / 48\right)\left(\partial_{x_{1}} S\right)^{2}+\phi(S)\right]
$$

where $\delta / \delta S$ indicates the functional derivative.

The TDGL equation (16) has two steady-state solutions except for a trivial solution $S=0$ : one is the uniform solution

$$
S\left(x_{1}, t_{1}\right)= \pm\left[6 V^{\prime}\left(2 V^{\prime} \tau-1\right) /\left|V^{\prime \prime \prime}\right|\right]^{1 / 2},
$$

and the other is the kink solution

$$
\begin{aligned}
S\left(x_{1}, t_{1}\right)= & \pm\left[6 V^{\prime}\left(2 V^{\prime} \tau-1\right) /\left|V^{\prime \prime \prime}\right|\right]^{1 / 2} \\
& \times \tanh \left[\left\{12\left(2 V^{\prime} \tau-1\right)\right\}^{1 / 2}\left(x_{1}-x_{10}\right)\right],
\end{aligned}
$$

where $x_{10}$ is a constant. Equation (18) represents the coexisting phase.

The coexisting curve is given by the condition

$$
\partial \phi / \partial S=0 \text { and } \partial^{2} \phi / \partial S^{2}>0
$$

From Eqs. (15) and (19), we obtain the coexisting curve in terms of the original parameters

$$
(\Delta x)_{c o}=h_{c}+\left[6 V^{\prime}\left(2 V^{\prime} \tau-1\right) /\left|V^{\prime \prime \prime}\right|\right]^{1 / 2} .
$$

The spinodal line is given by the condition

$$
\partial^{2} \phi / \partial S^{2}=0 .
$$

From Eqs. (15) and (21), we obtain the spinodal line in terms of the original parameters

$$
(\Delta x)_{\mathrm{sp}}=h_{c}+\left[2 V^{\prime}\left(2 V^{\prime} \tau-1\right) /\left|V^{\prime \prime \prime}\right|\right]^{1 / 2} .
$$

The critical point is given by the condition

$$
\partial \phi / \partial S=0 \text { and } \partial^{2} \phi / \partial S^{2}=0
$$

From Eqs. (15) and (23), we obtain the critical point in terms of the original parameters

$$
(\Delta x)_{c}=h_{c} \text { and } \quad 1 / \tau_{c}=2 V^{\prime} .
$$

Figure 1 shows the phase diagram between headway $\Delta x$ and inverse $1 / \tau$ of delay time where $h_{c}=5.0, V^{\prime}\left(h_{c}\right)=1$ and $V^{\prime \prime \prime}\left(h_{c}\right)=-2$ in Eq. (3). The solid curve indicates the coex-

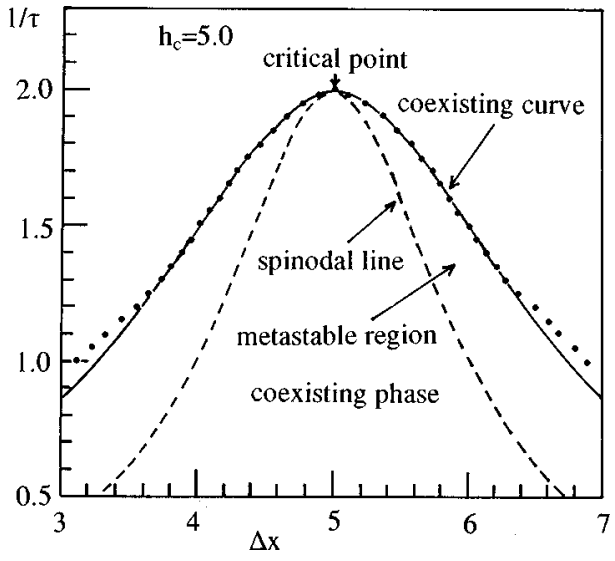

FIG. 1. Phase diagram in the $(\Delta x, 1 / \tau)$ plane, where $\Delta x$ is the headway, $1 / \tau$ is the inverse of delay time, the safety distance is $h_{c}$ $=5.0, V^{\prime}\left(h_{c}\right)=1$, and $V^{\prime \prime \prime}\left(h_{c}\right)=-2$. The critical point is given by $\Delta x=h_{c}=5.0$, and $1 / \tau=2.0$. The solid curve represents the coexisting curve given by Eq. (20). The dotted line represents the spinodal line given by Eq. (22). The full circles indicate the simulation result.

isting curve given by Eq. (20). The dotted line indicates the spinodal line given by Eq. (22). The critical point is given by $\Delta x=h_{c}=5.0$ and $1 / \tau=2.0$. In the region within the coexisting curve, the freely moving phase coexists with the congested phase. The intermediate regions between the coexisting curve and the spinodal line represent the metastable regions. The full circles indicate the simulation result of the coexisting phase boundaries explained lately in Sec. VI.

Generally, the jamming transition is the first-order phase transition below the critical point. The metastability observed in the traffic flow models corresponds to the spinodal decomposition in the conventional first-order phase transition.

\section{MODIFIED KdV EQUATION}

We derive the modified $\mathrm{KdV}$ equation from Eqs. (1) and (3). Then, we show the connection between the modified $\mathrm{KdV}$ equation and the TDGL equation. We consider the slowly varying behavior at long wavelengths near the critical point. We extract slow scales for space variable $n$ and time variable $t$. For $0<\varepsilon \ll 1$, we define the slow variables $X$ and $T$

$$
X=2 \varepsilon\left(n+V^{\prime} t\right) \text { and } T=\varepsilon^{3} V^{\prime} t / 3 \text {, }
$$

where $\varepsilon^{2}=2 V^{\prime} \tau-1$.

We set headway $\Delta x_{n}(t)$ as

$$
\Delta x_{n}(t)=h_{c}+\varepsilon\left(V^{\prime} /\left|V^{\prime \prime \prime}\right|\right)^{1 / 2} r(X, T) .
$$

By inserting Eq. (26) into Eq. (6) and expanding to fifth order of $\varepsilon$, we obtain the regularized equation

$$
\partial_{T} r-\partial_{X}^{3} r+\partial_{X} r^{3}=-\varepsilon M[r]
$$

where

$$
M[r]=6 \partial_{X}^{2} r+\partial_{X}^{4} r-\partial_{X}^{2} r^{3}
$$


Equation (27) is the modified $\mathrm{KdV}$ equation with an $O(\varepsilon)$ correction term on the right-hand side. Equation (27) is consistent with Eq. (12). We note that hereafter all derivatives of the optimal velocity are evaluated at $\Delta x=h_{c}$.

Let us find the propagating solution of Eq. (27) with constant velocity $c$ by setting $r(X, T)=r(X-c T)$. First, we ignore the $O(\varepsilon)$ terms in Eq. (27) and get the modified $\mathrm{KdV}$ equation, which has the kink solution

$$
r_{0}(X-c T)=c^{1 / 2} \tanh \left[(c / 2)^{1 / 2}(X-c T)\right] .
$$

Next, assuming $r=r_{0}+\varepsilon r_{1}$, we take into account the $O(\varepsilon)$ correction and get the equation for $r_{1}$ as

$$
L r_{1}=M\left[r_{0}\right],
$$

where

$$
L=c \partial_{X}+\partial_{X}^{3}-3 r_{0}^{2} \partial_{X}-3 \partial_{X} r_{0}^{2}
$$

To determine the selected value of the propagation velocity $c$ for the kink solution (29), we consider the solvability condition for Eq. (30):

$$
\left(\Phi_{0}, M\left[r_{0}\right]\right) \equiv \int_{-\infty}^{+\infty} d X \Phi_{0} M\left[r_{0}\right]
$$

where $\Phi_{0}$ is the zeroth eigenfunction of the adjoint operator $L^{\dagger}$ :

$$
L^{\dagger} \Phi_{0}=0, \quad L^{\dagger}=-c \partial_{X}-\partial_{X}^{3}+3 r_{0}^{2} \partial_{X}
$$

Fortunately, we find that the zeroth-order solution $r_{0}$ itself satisfies Eq. (33) and can choose $\Phi_{0}=r_{0}$. Performing the integration, we obtain the selected velocity as

$$
c=6 \text {. }
$$

This value is different from the result of the differential equation model obtained by Komatsu and Sasa [32]. Near the critical point, we obtain the propagating kink solution:

$$
\begin{aligned}
\Delta x_{n}(t)= & h_{c} \pm\left[6 V^{\prime}\left(2 V^{\prime} \tau-1\right) /\left|V^{\prime \prime \prime}\right|\right]^{1 / 2} \\
& \times \tanh \left[\left\{12\left(2 V^{\prime} \tau-1\right)\right\}^{1 / 2}\left\{n+V^{\prime}\left(2-2 V^{\prime} \tau\right) t\right\}\right] .
\end{aligned}
$$

The propagating velocity $v_{p}$ of jam (kink) is given by

$$
v_{p}=V^{\prime}\left(2-2 V^{\prime} \tau\right) \text {. }
$$

The kink solution (35) obtained from the modified $\mathrm{KdV}$ equation agrees with Eq. (18) by the TDGL equation.

The traffic jam seems to be static from the point of view of the backward moving frame with velocity (36). Thus, the jamming transition can be described by both TDGL equation (16) with a nontraveling solution and modified $\mathrm{KdV}$ equation (27) with a propagating solution.

\section{LINEAR STABILITY ANALYSIS}

We apply the linear stability theory to our model. We consider the stability of a uniform traffic flow. The uniform traffic flow is defined by such a state that all cars move with

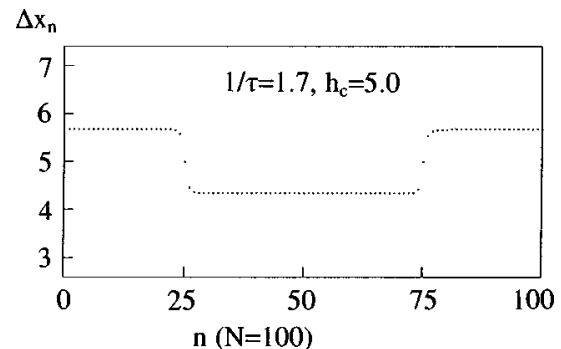

FIG. 2. Typical plot of the headway against numbered car $n$ for total car number 100 , car density $\rho=0.2$, safety distance $h_{c}=5.0$, and inverse $1 / \tau=1.7$ of delay time. The region with the short headway indicates the density wave (traffic jam), which propagates backward.

constant headway $h$ and optimal velocity $V(h)$. The solution of the uniform steady state is given by

$$
x_{n, 0}(t)=h n+V(h) t \quad \text { with } \quad h=L / N,
$$

where $N$ is the number of cars, $L$ is the system size, and $h$ is the car spacing (identical headway).

Let $y_{n}(t)$ be small deviations from the uniform solution $x_{n, 0}(t): x_{n}(t)=x_{n, 0}(t)+y_{n}(t)$. Then, the linearized equation is obtained from Eq. (1),

$$
d y_{n}(t+\tau) / d t=V^{\prime}(h) \Delta y_{n}(t)
$$

where $V^{\prime}(h)$ is the derivative of optimal velocity $V(\Delta x)$ at $\Delta x=h$.

By expanding $y_{n}(t)=Y \exp (i k n+z t)$, one obtains

$$
z e^{z \tau}=V^{\prime}(h)\left(e^{i k}-1\right) .
$$

Let us derive the long wave expansion of $z$, which is determined order by order around $i k \sim 0$,

$$
\begin{aligned}
z & =z_{1} i k+z_{2}(i k)^{2}+z_{3}(i k)^{3}+z_{4}(i k)^{4}, \\
z_{1} & =V^{\prime}, \\
z_{2} & =-V^{\prime}\left(2 V^{\prime} \tau-1\right) / 2, \\
z_{3} & =V^{\prime} / 6-V^{\prime 3} \tau^{2} / 2+V^{\prime 2} \tau\left(2 V^{\prime} \tau-1\right),
\end{aligned}
$$

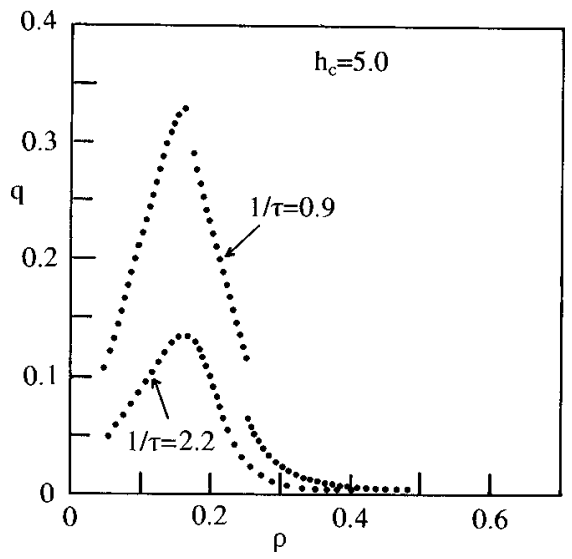

FIG. 3. Fundamental diagram of flow $q$ against density $\rho$. There are two kinds of data points for $1 / \tau=2.2$ and $1 / \tau=0.9$. For $1 / \tau$ $=0.9$ below the critical point, there are two discontinuous points characterizing the transitions. 


$$
\begin{aligned}
z_{4}= & V^{\prime} / 24-V^{\prime 2} \tau / 3+5 V^{\prime 4} \tau^{3} / 6 \\
& -7 V^{\prime 3} \tau^{2}\left(2 V^{\prime} \tau-1\right) / 4-V^{\prime 2} \tau\left(2 V^{\prime} \tau-1\right)^{2} / 4
\end{aligned}
$$

where $V^{\prime}=V^{\prime}(h)$.

If $z_{2}<0$, the uniform traffic flow is unstable. We obtain the stability condition $\tau<1 /\left[2 V^{\prime}(h)\right]$. When a small disturbance is added to the uniform flow (with a constant headway and the optimal velocity) satisfying the above condition, its uniform flow is always stable. We obtain the neutral stability condition

$$
\tau=1 /\left[2 V^{\prime}(h)\right] .
$$

By expanding $V^{\prime}(h)$ around the critical point $V^{\prime}\left(h_{c}\right): V^{\prime}(h)=V^{\prime}\left(h_{c}\right)+V^{\prime \prime \prime}\left(h_{c}\right)\left(h-h_{c}\right)^{2} / 2$ and replacing the expansion into Eq. (41), we obtain

$$
h-h_{c}= \pm\left[2 V^{\prime}\left(h_{c}\right)\left(2 V^{\prime}\left(h_{c}\right) \tau-1\right) /\left|V^{\prime \prime \prime}\left(h_{c}\right)\right|\right]^{1 / 2} .
$$

This is consistent with the spinodal line (22). The neutral stability line near the critical point agrees with the spinodal line.

\section{SIMULATION}

We perform a numerical simulation for our model described by Eqs. (1) and (3). Initially, cars are randomly distributed on the one-dimensional space with car density $\rho$ and initial velocity $v_{0}$. The boundary is periodic. In order to form a single jam, a hindrance is put at a point on the onedimensional space. We assume that when a car reaches the hindrance its car slows down instantly to low velocity. In time, a localized jam is formed just behind the hindrance. After the jam is formed, the hindrance is removed. The jam propagates backward with constant propagation velocity. Once the single jam is formed for a special range of density, the jam is stable and does not break up. The jam has a form of the kink-antikink. The stable jam occurs at an intermediate density. For low density, a jam disappears in time and all cars move freely with nearly maximal velocity. For high density, a jam also disappears in time and a congested uniform traffic flow appears. Thus, we can make a stable jam on the one-dimensional space for special values of density. We take the number of cars as 100-400. The safety distance is set as $h_{c}=5.0$. Figure 2 shows a typical plot of the headway against numbered car $n$ for total car number 100 , car density $\rho=0.2$, safety distance $h_{c}=5.0$, and inverse $1 / \tau=1.7$ of delay time. The profile of headway was obtained after a sufficiently long time. The stable density wave appears with the kink-antikink form. The density wave has a symmetric form. The region with the short headway indicates the traffic jam (density wave). The density wave propagates backward (from right to left) where cars move from left to right.
In Fig. 1, we show the plot of maximal headway $\Delta x_{\max }$ and minimal headway $\Delta x_{\min }$ against the inverse $1 / \tau$ of delay time where density $\rho=0.2$, safety distance $h_{c}=5.0$ and the total car number is 400 . The headway out of the jam is nearly constant but we choose the maximal value among them. Also, the headway within the jam is nearly constant but we choose the minimal value among them. One obtains the maximal and minimal headways when a density wave (traffic jam) occurs. The full circular points indicate the maximal and minimal headways obtained from simulation. For a special range of density, a jam appears. When a jam occurs, the maximal and minimal headways result in the same values for a constant value of $\tau$. The simulation result agrees with the coexisting curve obtained from the theory. There is a critical point. It is given by $h_{c}=5.0$ and $(1 / \tau)_{c}=1.95 \pm 0.05$. When $1 / \tau$ is larger than $(1 / \tau)_{c}$, no density wave appears.

We calculate the fundamental diagram. The fundamental diagram is obtained for an initial random configuration of cars without the hindrance. Figure 3 shows the fundamental diagram of flow $q$ against density $\rho$. Two kinds of data points for $1 / \tau=2.2$ and $1 / \tau=0.9$ are indicated in Fig. 3. For $1 / \tau$ $=0.9$ below the critical point, there are two discontinuous points characterizing the transitions in the fundamental diagram. Systems with $1 / \tau$ below the critical point depicted in Fig. 3 differ from systems with $1 / \tau$ above the critical point by displaying no transition. The gaps appearing in the fundamental diagram below the critical point are due to the metastability $[33,34]$. The phenomenon is similar to that observed by Krauss, Wagner, and Gawron [33].

\section{SUMMARY}

We presented the formulation of the thermodynamic theory for the jamming transition in traffic flow. We derived the TDGL equation from the car-following model, using the perturbation method. We showed that the order parameter is given by the headway and the inverse $1 / \tau$ of delay time corresponds to temperature in the conventional phase transition. We found the thermodynamic potential described by the headway and $1 / \tau$ for the traffic flow. We showed that the coexisting curve, the spinodal line, and the critical point are described by the derivatives of the potential, similarly to the conventional phase transitions and critical phenomena. We also carried out the numerical simulation. We compared the theoretical result with the simulation result. We showed that the coexisting curve agrees with the simulation. We derived the modified $\mathrm{KdV}$ equation from the car-following model and showed the connection between the TDGL equation and the modified $\mathrm{KdV}$ equation. We showed that the neutral stability line is consistent with the spinodal line. We proved that the jamming transition can be described exactly in terms of terminology of the phase transitions and critical phenomena.
[1] Traffic and Granular Flow, edited by D. E. Wolf, M. Schreckenberg, and A. Bachem (World Scientific, Singapore, 1996); D. Helbing, Verkehrsdynamik (Springer, Berlin, 1997).

[2] G. F. Newell, Oper. Res. 9, 209 (1961).
[3] G. B. Whitham, Proc. R. Soc. London, Ser. A 428, 49 (1990).

[4] M. Bando, K. Hasebe, A. Nakayama, A. Shibata, and Y. Sugiyama, Phys. Rev. E 51, 1035 (1995); M. Bando, K. Hasebe, K. Nakanishi, A. Nakayama, A. Shibata, and Y. Sugiyama, J. 
Phys. I (France) 5, 1389 (1995).

[5] K. Nagel and M. Schreckenberg, J. Phys. I (France) 2, 2221 (1992).

[6] M. Schreckenberg, A. Schadschneider, K. Nagel, and N. Ito, Phys. Rev. E 51, 2329 (1995).

[7] K. Nagel and H. J. Herrmann, Physica A 199, 254 (1993).

[8] G. Csanyi and J. Kertestz, J. Phys. A 28, 427 (1995).

[9] S. C. Benjamin, N. F. Johnson, and P. M. Hui, J. Phys. A 29, 3119 (1996).

[10] M. Takayasu and H. Takayasu, Fractals 1, 860 (1993).

[11] M. Fukui and Y. Ishibashi, J. Phys. Soc. Jpn. 66, 385 (1997).

[12] A. Schadschneider and M. Schreckenberg, Ann. Phys. (Leipzig) 6, 541 (1997).

[13] H. Emmerich and E. Rank, Physica A 216, 435 (1995).

[14] O. Biham, A. A. Middleton, and D. A. Levine, Phys. Rev. A 46, R6124 (1992).

[15] T. Nagatani, Phys. Rev. E 48, 3290 (1993).

[16] J. A. Cuesta, F. C. Matinez, J. M. Molera, and A. Sanchez, Phys. Rev. E 48, 4175 (1993).

[17] K. H. Chung, P. M. Hui, and G. Q. Gu, Phys. Rev. E 51, 772 (1995).

[18] I. Prigogine and R. Herman, Kinetic Theory of Vehicular Traffic (Elsevier, New York, 1971).

[19] S. L. Paveri-Fontana, Transp. Res. 9, 225 (1975).

[20] D. Helbing, Phys. Rev. E 53, 2366 (1996).

[21] D. Helbing, Physica A 233, 253 (1996).
[22] T. Nagatani, Physica A 237, 67 (1997).

[23] E. Ben-Naim, P. L. Krapivsky, and S. Redner, Phys. Rev. E 50, 822 (1994).

[24] T. Nagatani, Phys. Rev. E 51, 922 (1995).

[25] J. Krug and P. A. Ferrari, J. Phys. A 29, L465 (1996).

[26] M. R. Evans, Europhys. Lett. 36, 13 (1996).

[27] B. S. Kerner and P. Konhauser, Phys. Rev. E 48, 2335 (1993).

[28] B. S. Kerner, P. Konhauser, and M. Schilke, Phys. Rev. E 51, 6243 (1995).

[29] B. S. Kerner and H. Rehborn, Phys. Rev. E 53, R1297 (1996).

[30] B. S. Kerner and H. Rehborn, Phys. Rev. Lett. 79, 4030 (1997).

[31] D. A. Kurtze and D. C. Hong, Phys. Rev. E 52, 218 (1995).

[32] T. Komatsu and S. Sasa, Phys. Rev. E 52, 5574 (1995).

[33] S. Krauss, P. Wagner, and C. Gawron, Phys. Rev. E 55, 5597 (1997).

[34] R. Barovic, L. Santen, A. Schadschneider, and M. Schreckenberg (unpublished).

[35] T. Nagatani, Physica A 246, 460 (1997).

[36] K. Kawasaki, Ann. Phys. (N.Y.) 154, 319 (1984).

[37] J. D. Gunton, M. San Miguel, and P. S. Sahni, in Phase Transitions and Critical Phenomena, edited by C. Domb and J. L. Lebowitz (Academic, New York, 1983), Vol. 8, p. 267.

[38] M. C. Cross and P. C. Hohenberg, Rev. Mod. Phys. 65, 851 (1993). 